Cahiers « Mondes anciens »

MONDES

ANCIENS

Histoire et anthropologie des mondes anciens

$1 \mid 2010$

Écritures rituelles

\title{
Le pouvoir de l'écriture dans la magie
}

\section{Attilio Mastrocinque}

\section{(2) OpenEdition}

\section{Journals}

Édition électronique

URL : http://journals.openedition.org/mondesanciens/168

DOI : 10.4000/mondesanciens. 168

ISSN : 2107-0199

Éditeur

UMR 8210 Anthropologie et Histoire des Mondes Antiques

\section{Référence électronique}

Attilio Mastrocinque, «Le pouvoir de l'écriture dans la magie », Cahiers "Mondes anciens » [En ligne], 1 | 2010, mis en ligne le 17 mai 2013, consulté le 19 avril 2019. URL : http://journals.openedition.org/ mondesanciens/168; DOI : 10.4000/mondesanciens.168

Ce document a été généré automatiquement le 19 avril 2019

\section{(c) (i) $\odot$}

Les Cahiers «Mondes Anciens » sont mis à disposition selon les termes de la licence Creative Commons Attribution - Pas d'Utilisation Commerciale - Pas de Modification 4.0 International. 


\title{
Le pouvoir de l'écriture dans la magie
}

\author{
Attilio Mastrocinque
}

1 L'écriture peut constituer un rite sacré parce qu'on écrit des noms, des formules ou des signes sacrés, parce qu'on écrit sur des images divines ou des objets sacrés, parce que ceux qui écrivent sont des opérateurs du sacré en fonction, enfin parce qu'une écriture particulière doit être tracée selon une modalité rituelle.

2 Nous envisageons ici le cas d'une écriture performative employée dans la magie. Est "performative» une parole qui agit par le fait même qu'on la prononce. Ainsi, par exemple, quand je dis : "Je te bénis », tu es béni par ce fait même que la formule est prononcée. L'écriture aussi peut être performative. Dans le moment même où j'écris, une action se produit du fait même que j'ai tracé les mots selon certaines modalités précises. Un tel art était employé dans la magie antique : il unissait le sens des mots à des formes géométriques que l'on conférait au tracé de ces mots. Ensemble, forme géométrique et contenu donnaient aux mots une force particulière, propre à l'écriture avant toute prononciation.

3 Le Testament de Salomon, une œuvre de magie judaïsante de la première époque impériale, décrit le pouvoir des démons et les moyens de les exorciser et de les contrôler. Le démon Rhyx se présente en disant: "Je m'appelle aussi Rhyx Achôneôth. Je cause le mal au pharynx et aux amygdales. Si quelqu'un écrit sur une feuille de lierre $\Lambda$ EIKOYPГOC, en forme de grappe ANAXSPIC, tu t'en vas $»^{1}$.

4 Évidemment il fallait écrire :

$\Lambda$ EIKOYPГOC
ANAX $\Omega$ PIC
NAX $\Omega$ PI
AX $\Omega$ P
X $\Omega$

5 Ou plutôt, étant donné que le nombre de lettres est pair :

$\Lambda$ EIKOYРГОС

ANAXSPIC

NAX $\Omega$ PIC 


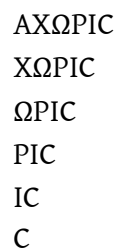

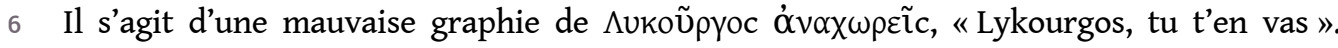
Lykourgos est l'ennemi de Dionysos, toujours représenté en train de couper la vigne avec sa hache.

7 Un papyrus de la Bibliothèque Laurentiana de Florence conserve une amulette du même type $^{2}$ :

СТАФУАОТОМОС
ТАФУАОТОМОС
АФУАОТОМОС
ФАОТОМОС
УАООМОС
АОТОМОС
ОТОМОС
ТОМОС
ОМОС
МОС
OC
С

On aura reconnu le double sens du grec staphulê et du latin $u v a^{3}$, qui se donne à lire sur un double registre : à la fois grappe de raisin et uvule (c'est-à-dire la luette, et par contiguïté les amygdales) ; et le mot lui-même tel qu'il est dessiné en forme de grappe sur l'amulette.

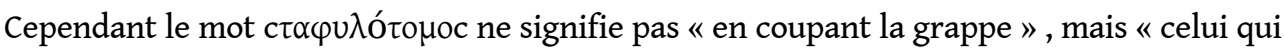
coupe la grappe ", c'est à dire, précisement, Lykourgos. L'idée qui préside à cette formule magique est que Lykourgos est une menace pour la vigne, et que pour cette raison la maladie qui rend l'uvule ou les amygdales semblables à une grappe de raisin s'enfuit au

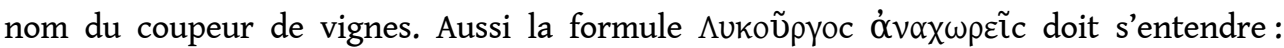
"Voici Lykourgos, et toi, maladie en forme de grappe, tu t'en vas ».

9 Une gemme en lapis-lazuli de la Bibliothèque Nationale de Paris ${ }^{4}$ est gravée d'une semblable inscription :

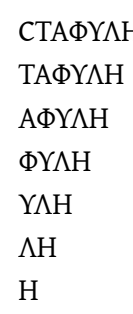

Sur l'autre face de la gemme est gravée l'image d'une grappe.

11 Ce type d'amulette fait partie d'une série de remèdes qui ont recours à des figures de la mythologie grecque pour combattre des maladies en invoquant certaines caractéristiques mythiques des héros.

Si l'on suit la piste ici découverte, il devient possible de reconnaître la vraie nature d'un papyrus magique ${ }^{5}$, connu depuis longtemps, dans lequel on lit :

$Г О Р Г \Omega Ф \Omega N A C$

ОРГ $\Omega \Phi \Omega N A C$ 


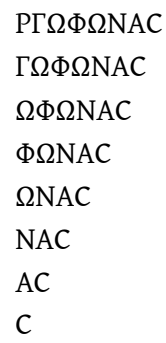

Ces lettres ainsi disposées sont accompagnées de la formule suivante :

Je vous adjure, au nom du nom saint, de guérir Dionysos, dit aussi Anys, mis au monde par Héraklia, de tout frisson et fièvre, soit journalière soit intermittente, le jour ou la nuit, soit quartaine. Maintenant, maintenant, maintenant! Vite, vite, vite!

Dans la récente traduction anglaise des papyrus magiques ${ }^{6}$, John Scarborough suppose que Gorgophonas est un accusatif pluriel féminin, pouvant se référer à Athéna, qui tua la Gorgone. En réalité, celui qui tua la Gorgone fut Persée, et non pas Athéna.

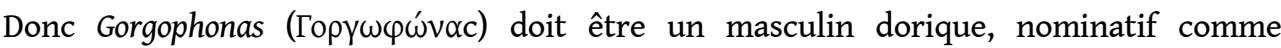

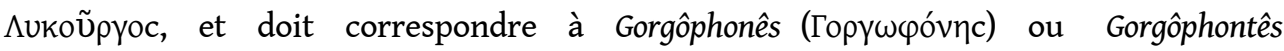

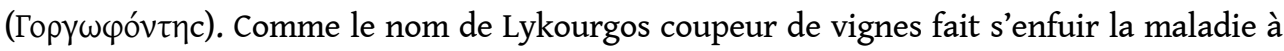
forme de grappe de raisin, ainsi le nom de Persée, tueur de la Gorgone, fait s'enfuir le démon de la malaria ${ }^{7}$.

Une gemme magique très fameuse, conservée à Saint-Pétersbourg, représente Persée

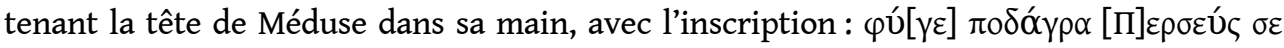
$\delta \iota^{\prime} \chi_{1}{ }^{8}:$ « Fuis, podagre, Persée te poursuit ».

17 On connaît une petite série de gemmes magiques ${ }^{9}$ en hématite, qui étaient portées pour combattre les pertes de sang provoquées soit par des hémorragies, soit par des menstruations irrégulières, soit par des ulcères. Elles représentent le dieu Arès accompagné de l'inscription :

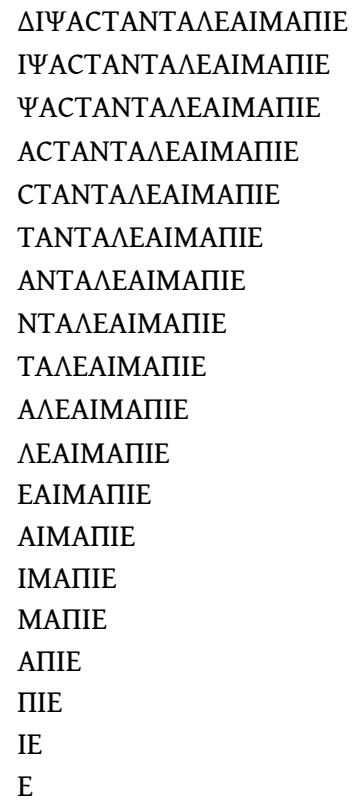

18 L'inscription signifie «Tantale, tu as soif : bois du sang! » Le sens de ce texte a été éclairci par Alphonse Barb ${ }^{10}$ : «Le sang va se retirer d'un seul coup, aussitôt que le malheureux damné tentera de le boire ». Le recours à la figure de Tantale est attesté aussi par des recettes médicinales ou de tradition populaire ${ }^{11}$. 
19 Nous nous bornons aux amulettes qui ont recours à des figures géométriques de ce type, tout en rappelant qu'il existe d'autres amulettes semblables, évoquant Éole ou Héraklès contre les maladies. On peut aisément supposer que la série des amulettes médicinales invoquant des personnages du mythe grec au moyen de formules inscrites en triangles remontent au même manuel de magie ${ }^{12}$.

20 Le nom ou la phrase sont efficaces contre les maladies, c'est-à-dire contre les démons des maladies, parce qu'ils sont écrits en triangle. Les mots en triangle peuvent subjuguer les démons; ils ont une puissance qui leur vient de leur nature divine et du fait qu'ils additionnent les pouvoirs de chacune des parties qui les composent. Parfois les triangles

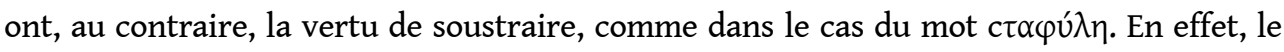
nom d'une maladie ou d'un élément qui évoque la maladie s'écrit de façon que le décroître du mot efface progressivement la maladie même ${ }^{13}$.

21 Les papyrus magiques offrent à leur tour quantité de formules inscrites en triangle ou en d'autres formes géométriques, destinées soit à chasser les maladies, soit à soumettre des démons dans une intention maléfique, comme dans le cas des défixions.

22 Parmi les formules en triangles nous distinguerons les palindromes et le recours aux sept voyelles, comme dans la recette du papyrus magique XCVIII, appelant Sérapis à libérer (de maladies) une certaine Artémidora et prescrivant un triangle ainsi composé :

A

EE

$\mathrm{HHH}$

IIII

00000

YMYY

$\Omega \Omega \Omega \Omega \Omega \Omega \Omega$

23 On rencontre des triangles ou des séries de triangles du même ordre ${ }^{14}$ dans certaines formules d'attraction, quelquefois très aggressives, comme celle du papyrus magique $\mathrm{XXXVI}^{15}$ où apparaissent trois figures géométriques, dont la troisième est désignée comme un bathron, une « échelle » :

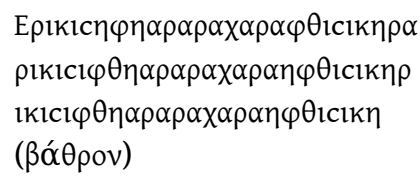

24 Le cas le plus extrême est celui du papyrus magique XIX, qui servait pour contraindre et subjuguer l'âme d'un mort et l'envoyer plier la volonté d'une femme au désir amoureux du bénéficiaire de la magie. Ce papyrus plein de séries géométriques a été trouvé replié dans la bouche d'un mort.

Le même nom magique Eulamô (" éternité ») en formation triangulaire réapparaît sur une pierre gravée destinée à susciter une attraction amoureuse ${ }^{16}$, ainsi que sur une défixion à l'encontre des cochers du cirque, découverte à Rome ${ }^{17}$.
EYAAM $\Omega$
$\Upsilon \Lambda \mathrm{AM} \Omega$
$\Lambda \mathrm{AM} \Omega$
$\mathrm{AM} \Omega$
$\mathrm{M} \Omega$
$\Omega$

Étant donné que le tracé de ces figures géométriques avait le pouvoir de soumettre les démons, on l'employait pour obtenir un démon assistant. La procédure est décrite dans le 
PGM I ${ }^{18}$ dans lequel deux formations de voyelles sont dessinées et appelées $k \lambda i ́ \mu \alpha \tau \alpha$, terme énigmatique qui doit probablement se comprendre comme k $\lambda \hat{i} \mu \alpha \kappa \varepsilon c$, «échelles " ${ }^{19}$, définissant la formation triangulaire que nous avons mentionnée ci-dessous.

\begin{tabular}{|l|l|}
\hline A & $\Omega \Omega \Omega \Omega \Omega \Omega \Omega$ \\
EE & IYYY \\
HHH & OOOOO \\
IIII & III \\
OOOOO & HHH \\
YYYY & EE \\
$\Omega \Omega \Omega \Omega \Omega \Omega \Omega$ & A \\
\hline
\end{tabular}

On ne saurait dire quelle était l'origine précise de cette géométrie de la parole magique. Un papyrus magique ${ }^{20}$ prescrit de graver une lamelle d'une série de charaktêres en étant soi-même en état de pureté, les poignets enguirlandés de fleurs, après avoir offert de l'encens. La force de cette étrange méthode d'écriture rituelle résidait dans les figures géométriques réalisées. Il est possible que le mot « échelle » se soit référé seulement à la forme du triangle, pourvu de marches formées par le décalage des lettres.

Dans les papyrus magiques on lit que les formations de lettres ou de mots en triangles sont dites « ailes » ou « cœurs » (pteruges, pterugômata, kardiai ${ }^{21}$ ), ou, en cas de formation en rectangle, plinthion ${ }^{22}$, «briquette». Probablement s'agit-il simplement d'une ressemblance triviale entre les formes des mots et celles des ailes, des cœurs ou des briques : ces noms seraient donc tout à fait conventionnels. Sans doute la grappe destinée à guérir l'uvule est-elle une exception ${ }^{23}$, car elle se trouve présenter la même forme que la maladie.

Le principe inspirateur des triangles est l'exaltation du mot magique à travers sa multiplication. Franz Dornseiff ${ }^{24}$ a remarqué que la formation en triangle remontait à une tradition des Pythagoriciens, qui concevaient la Tétraktys comme la génération du 10 par sa mère, le 4 , à travers l'addition de $1+2+3+4^{25}$. On représentait parfois la Tétraktys sous forme de triangle :

$\circ$

000

0000

Ce type de figure géométrique et mathématique intéressait aussi les Platoniciens, jusqu’à Damascius $^{26}$, selon qui la chatte calculait les 28 formes de la lumière de la lune, parce qu'elle engendrait 28 petits, 7 à la première portée, 6 à la deuxième, 5 à la troisième, 4 à la quatrième, à la cinquième, 2 à la sixième, 1 à la septième, avec un schéma en triangle de 28 unités :

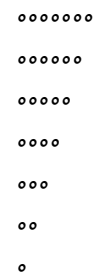

31 Roy Kotansky ${ }^{27}$ a récemment étudié une lamelle-phylactère d'Éphèse portant une série de 17 voyelles dans une formation triangulaire de 17 lignes, qui donne un total de 153 voyelles. Kotansky a identifié dans le commentaire de saint Augustin à l'évangile de Jean ${ }^{28}$ 
la clé d'interprétation de cette figure. Augustin écrit que 153 est la somme de : $1+2+3+4+5$ $+6+7+8+9+10+11+12+13+14+15+16+17$.

L'« aile » de l'amulette d'Éphèse se référait à ce type d'addition. La logique de ce type de tracé écrit peut se comparer à celle qui présidait à la création de noms divins à valeurs numériques, procédant de l'addition d'un nombre et de ses multiples par dix, cent et mille. On s'efforçait de créer des mots qui eussent une valeur de 99,9999 , ou 888,666 , $3663^{29}$.

Les formations géométriques magiques traitaient donc les mots comme des additions. EYLAMÔ est l'addition de Ô+MÔ+AMÔ+LAMÔ+YLAMÔ+EYLAMÔ.

Notons que si le pouvoir des triangles réside dans l'addition ou dans la soustraction des lettres, celui des «briques " réside dans leur multiplication. Les «briques » présentent quelquefois une dynamique hélicoïdale, comme dans le cas des séries des sept voyelles, que l'on écrit sept fois en les décalant d'une voyelle à chaque ligne ${ }^{30}$. Chaque voyelle correspond à la note musicale d'une planète, et la circulation des voyelles au sein de la série symbolise le mouvement circulaire des planètes. On lisait par exemple dans la «brique » suivante la série canonique des voyelles, dans le sens horizontal et dans le sens vertical :

AEHIOY $\Omega$

EHIOY $\Omega A$

HIOY $\Omega A E$

IOY $\Omega A E H$

OYSAEHI

Y $\triangle A E H I O$

SAEHIOY

L'écriture des mots magiques a sa dynamique interne dans ce sens aussi qu'elle agit par des figures, des changements, des inversions, des coupures, opérés sur le nom de la personne ou du démon qui se trouve inscrit en position contiguë. En 1914 Armand Delatte ${ }^{31}$, en décrivant les palindromes magiques, que les Grecs appelaient anagrammata ${ }^{32}$, écrivait: "Les formules anagrammatiques, qui sont faites pour des inscriptions circulaires et dont le modèle est le serpent ourobore, sont une image du cours du Soleil ». Un palindrome, comme le fameux ABLANATHANALBA, parce qu'il se lisait dans les deux sens, pouvait être facilement écrit en forme de grappe. On le retrouve aussi en forme de cercle, comme dans une gemme d'attraction amoureuse de la collection De Clercq ${ }^{33}$. On y lit le nom FAVSTINA encerclé par le palindrome ABLANATHANALBA. Ce mot écrit en cercle est comme un anneau qui emprisonne et donc contraint la femme dont le nom est encerclé. Un papyrus magique décrit le rite par lequel on contraignait une personne à faire ou à ne pas faire une chose ${ }^{34}$. On prenait un morceau de papyrus hiératique ou une lamelle de plomb et un anneau de fer, avec une plume on traçait les cercles extérieur et intérieur de l'anneau sur le papyrus ou la lamelle. Dans l'anneau dessiné on écrivait une longue formule magique, dans le cercle intérieur on dessinait un rectangle de mots magiques et on écrivait: "Que telle chose ne soit pas faite tant que cet anneau restera enseveli ». On repliait le papyrus ou la lamelle sur l'anneau, on les liait et on les plaçait dans une source abandonnée ou dans la tombe d'un mort prématuré. Le même papyrus conserve encore le dessin d'une défixion de ce type et présente le palindrome :

IAE $\Omega B A Ф P E N E M O Y N O \Theta I \Lambda A P I K P I Ф I A E Y E A I Ф I P K I P A \Lambda I \Theta O N Y O M E N E P Ф A B \Omega E A I$.

Dans les papyrus magiques cette formule est présentée comme équivalente du nom du dieu juif ${ }^{35}$ ainsi que du serpent ouroboros, qui « mange sa queue $»^{36}$. 
Les tracés d'écriture magique ici présentés mettent en évidence un mécanisme fréquemment à l'œuvre les opérations de magie : le pouvoir de ces formules réside dans une forme de court-circuit. Tandis que dans le cercle le mot palindrome tourne indéfiniment sur lui-même, dans le triangle le mot additionne ou, parfois, soustrait ses éléments, dans le carré il se multiplie par lui-même. Cet emploi mathématique et géométrique des lettres ou des mots inscrits trahit l'influence de spéculations pythagoriciennes (certains y verraient volontiers encore des influences chaldéennes), c'est-à-dire des théoriciens de la mystérieuse nature géométrique et mathématique de toutes les choses, des êtres vivants et des dieux.

\section{BIBLIOGRAPHIE}

BARB Alphonse, 1952, « Bois du sang, Tantale », Syria, 29, p. 271-84.

BONNER Campbell, 1950, Studies in Magical Amulets, Ann Arbor.

Colson F. H., 1914, « Triangular Numbers in the New Testament ", Journal of Theological Studies, 16, p. 67-76.

DANIEL Robert W. \& MALTOMINI F., 1979, « Una gemma magica contro l'infiammazione dell'ugola », Zeitschrift für Papyrologie und Epigraphik, 78, p. 93-4.

DANiel Robert W. \& MALTOMINI F., 1990, Supplementum magicum, I (P. Colon. 16), Opladen.

De RIDDER André, 1911, Collection de Clercq. Catalogue. VII : Les bijoux et les pierres gravées, Paris.

DELATTE Armand, 1914, «Études sur la magie grecque », Musée Belge, 18, p. 5-96

DELATTE Armand \& DERCHAIN Philippe, 1964, Les intailles magiques gréco-égyptiennes de la Bibliothèque Nationale, Paris.

DEONNA Walter, 1944, «ABRA, ABRACA : La croix-talisman de Lausanne », Genava, 22, p. 131-3.

DORNSEIFF Franz, 1925, Das Alphabet in Mystik und Magie, Leipzig-Berlin, $1925^{2}$.

ERNST Ulrich, 1991, Carmen figuratum, Köln.

FESTUGIÈRE André-Jean, 1975, Études d'histoire et de philologie, Paris.

- 1951, « Amulettes magiques », Classical Philology, 46, p. 86-9.

FRANKFURTER David, 1994, « The Magic of Writing and the Writing of Magic - the Power of the Word in Egyptian and Greek Traditions ", Helios, 21, p. 189-221.

GMA : cf. KOTANSKY, 1994

KOTANSKY Roy D., 1994, Greek Magical Amulets : the Inscribed Gold, Silver, Copper and Bronze Lamellae, I : Published Texts of Known Provenance (Papyrologica Colonensia 22.1), Opladen.

KRAUSS Friedrich, 1973, Das Theater von Milet (Milet, IV. 1), Berlin.

LENZ C., 1954, « Carmina figurata », Realkexikon für Antike und Christentum 2, p. 910-12. 
MCELENEy N. J., 1977, « 153 Great Fishes (John 21,11)-Gematrical Atbash », Biblica, 58, p. 411-7. MARTINEZ David G., 1991, P. Michigan XVI : A Greek Love Charm from Egypt (P. Mich 757), Atlanta. MASTROCINQUE Attilio, 2000, « Studi sulle gemme gnostiche. VIII. "Bevi sangue, Tantalo" », Zeitschrift für Papyrologie und Epigraphik, 130, p. 137-8.

- 2002, « Metamorfosi di Kronos su una gemma di Bologna », in A. Mastrocinque (éd.) Gemme gnostiche e cultura ellenistica. Atti dell'incontro di studio Verona, $22-23$ ottobre 1999, Bologna.

- 2005, From Jewish Magic to Gnosticism, Tübingen.

-2008, Sylloge gemmarum gnosticarum, II, Istituto Poligrafico dello Stato (collana Bollettino di numismatica).

MICHEL Simone, 2001, Die magischen Gemmen im Britischen Museum, London (P. \& H. Zazoff éd.).

NEVEROV Oleg J., 1976, Antique Intaglios in the Hermitage Collection, Leningrad.

PGM = Preisendanz Karl (éd.), Papyri Graecae Magicae, Leipzig-Berlin, 2 vol., 1973-1974² (1928-31²).

PGMT = Hans Dieter Betz (éd.), The Greek Magical Papyri in Translation*, 1, Chicago and London, 1993 $\left(1986^{1}\right)$.

PINTAUDI Rosario, 1959, Dai papiri della Biblioteca Medicea Laurenziana (Plaur. III), Firenze.

PRADEL Fritz, 1907, Griechische und süditalienische Gebete, RGVV III. 3, Giessen.

SAMBON Arthur, 1909, «La bague à travers les âges », Le Musée, 6, p. 112.

SCHмIDT C., 1931, 1934, Comptes rendus de Preisendanz, Göttingische Gelehrte Anzeigen, 193, p. 443

ss. ; 196, p. 177.

SEYRIG Henri, 1934, «Invidiae medici. 1. La faim de l'ibis et la soif de Tantale », Berytus, 1, p. 1-5.

Test. Salom. : Chester C. McCown (éd.), 1922, The Testament of Solomon, Leipzig.

VERSNEL Hendrik S., 1996, « Die Poetik der Zaubersprüke », in T. Schabert \& R. Brague (éd.), Die Macht des Wortes, München, p. 265-6.

WILCKEN Ulrich, 1901, Archiv für Papyrusforschung und verwandte Gebiete, 1, p. 422.

WOJACZEK Günter, 1969, Daphnis. Untersuchungen zur griechischen Bukolik, Meisenheim am Glan.

WORTMANN Dirk, 1968, Neue magische Texte, Bonner Jahrbücher, 168, p. 56-111.

wÜNSCH Richard, 1898, Sethianische Verfluchungstafeln aus Rom, Leipzig.

\section{NOTES}

1. Testament de Salomon XIX, 58.

2. PINTAUDI, $1959:$ nr. $59=$ PGM CXX.

3. Cf. Marcellus Empiricus, De médicina XIV. 25 ; PINTAUDI, 1959 : l.c.; KOTANSKY, in PGMT, p. 316 ; DANIEL \& MALTOMINI, 1979 : p. 93-4.

4. SAMBON, 1909 : p. 112 ; DANIEL \& MALTOMINI, cit.

5. PGM XVIIIb.

6. PGMT, p. 255.

7. Dans le Testament de Salomon XIII, 43-44 on retrouve le démon Obyzouth, c'est-à-dire Abyssos, qui n'est rien d'autre que la Gorgone elle-même. Son corps est invisible, recouvert de ténèbres, 
mais ses cheveux sont visibles et apparaissent dans leur nature sauvage. Elle admet que ses victimes préférées sont les enfants et les femmes, qu'elle s'attache aux yeux, à la bouche et à l'esprit des personnes. Elle a le pouvoir de glacer. Mais Salomon lui fait avouer qu'elle s'enfuit si l'on écrit son nom sur un papier quand une femme met au monde un enfant. L'ange qui s'oppose à elle est Raphaël.

8. NEVEROV, 1976 : n 143 a. PRADEL, 1907, p. 266-7, publie une amulette du moyen âge contre la quartaine, sur laquelle on écrivait : $K \alpha \delta \alpha \beta \rho \alpha \rho \circ \beta \beta \rho \alpha \kappa \alpha \delta \alpha \beta \rho \alpha \rho \circ \beta \beta \rho \alpha \kappa \alpha \delta \alpha \beta \rho \alpha \rho \alpha \kappa \varepsilon 1 \delta \alpha \beta \rho \alpha \kappa \alpha \delta \alpha$

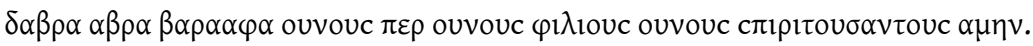

9. FESTUGIÈRE, 1975, p. 151 ; Id., 1951, p. 86-9 ; SEYRIG, 1934, p. 1-5 ; BONNER, 1950, p. 87-9 ; DELATTE \& DERCHAIN, 1964, nº 364 ; MASTROCINQUE, 2000, p. 137-8; MICHEL, 2001, nº 410.

10. BARB, 1952.

11. BARB, o.c.

12. Serenus Sammonicus prescrit, dans son Liber médicinales 51 (935 p. $48-9$ Pépin), d'écrire un triangle magique contre la fièvre qui revient tous les 2 jours et demi (hemitritaios) : «Inscribes chartae quod dicitur abracadabra saepius et subter repetes, sed detrahe summam et magis atque magis desint elementa figuris singula, quae semper rapies, et cetera figes, donec in angustum redigatur littera conum $:$ his lino nexis collum redimire memento $"$.

13. DANIEL \& MALTOMINI, 1979 : p. 93 ; VERSNEL, 1996 : p. 265-6.

14. Par exemple PGM XVIIa, 1-25 ; XXXVI, 115-133 ; XXXIX, 1-21.

15. XXXVI, 231-55.

16. MASTROCINQUE, $2008: \mathrm{n}^{\circ}$ Ro 21.

17. WÜNSCH, 1898 : p. 50-51, n. 48 ; cf. p. 41, n. 34.

18. $P G M I, 12-19$.

19. U. WILKEN, 1901, p. 422 : «Abstufungsfigur»; FRANKFURTER, 1994, p. 200 : «seven-layered»; KOTANSKY, 1994, p. 202 : « gradation-figure ».

20. PGM XIII, 1001-5. Selon FRANKFURTER, 1994, p. 205, les séries magiques de voyelles dérivent des hymnes vocaliques des prêtres égyptiens (Dem. Phal., Eloc.71), mais les auteurs anciens attribuent à Ostanès et à la tradition des Mages persans la théorie des sept sons vocaliques et des sept notes: Origène, Contre Celse VI. 22 ; Porphyre, dans Eusèbe, Préparation évangélique V. 14 = fr. 330 smith.

21. Pteryx, pterygoma : PGM II, 2 e 5 ; VII, 716 ; XIII, 903-4 ; kardiai : PGM II. 68 ; VII, 523 ; LXII sec. 1 , 82 ; MARTINEZ, 1991 : p. 13, cf. 105-111; dans PGM III, 60-70 la forme en cœur est dite équivalente à celle en grappe : $\kappa \alpha \rho \delta \_\alpha \kappa \tilde{\omega} c, \dot{\omega} c \beta$ ó $\rho \rho c$. Dans PGM XIII, 16 on parle d'un livre d'Hermès intitulé Pteryx.

22. IV, 1305 ; V, 348 ; 360 ; VII, 656.

23. Sur les noms des formations de mots et leur emploi : DANIEL \& MALTOMINI, 1990, p. 4-6 ; KOTANSKY, 1994, p. 202-5. Voir aussi DORNSEIFF, $1925^{2}$, p. 63-7 ; DEONNA, 1944, p. 131-3 ; LENZ, 1954, p. 910-12 ; WORTMANN, 1968, p. 104 ; WOJACZEK, 1969 : p. 59 ss. et n. 12 ; ERNST, 1991 : p. 33-45.

24. Alphabet, pp-58-59.

25. Philolaos, fr. A 11 Timpanaro Cardini ; Jamblique, Vie de Pythagore 18.82 (où la Tétraktys est définie comme l'harmonie des Sirènes) ; Pseudo-Jamblique, Theologoumena arithméticien 27-28; 86. 26. Damascius, Vie d'Isidore, dans Photius, 343 a2. Platon se servait de calculs du même genre

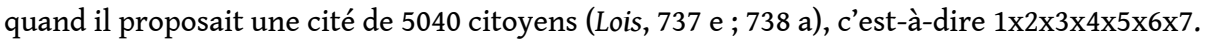

27. GMA I, n³7, p. 202-205.

28. In Johannis Evangelium CXXIII. 8.35-43 ; Corpus Christianorum XXXVI, 673 : le nombre 153 correspond au total des poissons de la pêche miraculeuse. Cf. COLSON, 1914 ; MCELENEY, 1977 : p. 411-7.

29. MASTROCINQUE, 2005 : p. 109-114.

30. Par exemple PGM X, 42-48; XIII, 905 ; XIXa sec. 18-23 ; KRAUSS, 1973, tab. 137 ; GMA I, n 9. 
31. DELATTE, 1914 : p. 28.

32. PGM X, 36-50.

33. DE RIDDER, 1911 : n³ 3470 ; cf. MASTROCINQUE, 2002 : p. 118.

34. PGM V, 304-69.

35. PGM IV, 3069.

36. PGM VII, 579-90 ; cf. aussi DELATTE \& DERChAIN, 1964, nr. 122 ; SGG I, nr. 191. Pour une interprétation de certaines parties de la formule en partant de l'égyptien, cf. SCHMIDT, 1931, p. 443 ss. ; 1934 , p. 177.

\section{RÉSUMÉS}

Les papyrus et les écrits magiques de l'Antiquité (époques impériale et tardive) témoignent d'un usage performatif et rituel de l'écriture, où les mots et les lettres disposés en triangles, en rectangles, en cercle, en vertu de la nature géométrique et mathématique de toutes choses, sont réputés guérir, protéger ou envoûter efficacement, selon que des lettres sont retranchées ou ajoutées (dans le triangle) à un mot initial, multipliées (dans le rectangle ou le carré), ou encore disposées en palindrome.

The magical papyri and other writings from imperial and late Antiquity attest a performative and ritual use of words and letters, arranged into triangles, rectangles, or circles. Because of the geometrical or mathematical nature of all things, these triangles or rectangles are supposed to be efficient for healing, protecting or casting a spell on someone. By removing or adding letters (in the case of the triangle), or by multiplying them (in the case of the rectangle), or by arranging them in a palindromic circular order, the magical power comes to effect.

\section{INDEX}

Keywords : demon, geometric words, magic, writing

Mots-clés : écriture, magie

\section{AUTEUR}

\section{ATTILIO MASTROCINQUE}

Université de Vérone 